\title{
RESEARCH
}

Open Access

\section{STAT3 activation by catalpol promotes osteogenesis-angiogenesis coupling, thus accelerating osteoporotic bone repair}

Liang Chen ${ }^{1,2}$, Ri-Yan Zhang ${ }^{3}$, Jun Xie ${ }^{1,2}$, Jia-Yi Yang ${ }^{4}$, Kang-Hao Fang ${ }^{1,2}$, Chen-Xuan Hong ${ }^{1,2}$, Rong-Bo Yang ${ }^{5}$, Najeeb Bsoul ${ }^{1}$ and Lei Yang ${ }^{1,2^{*}}$

\begin{abstract}
Background: Bone fracture repair has gained a lot of attention due to the high incidence of delayed union or even nonunion especially in osteoporotic patients, resulting in a dreadful impact on the quality of life. However, current therapies involve the costly expense and hence become unaffordable strategies for fracture recovery. Herein, developing new strategies for better bone repair is essential and urgent. Catalpol treatment has been reported to attenuate bone loss and promote bone formation. However, the mechanisms underlying its effects remain unraveled.
\end{abstract}

Methods: Rat bone marrow mesenchymal stem cells (BMSCs) were isolated from rat femurs. BMSC osteogenic ability was assessed using ALP and ARS staining, immunofluorescence, and western blot analysis. BMSC-mediated angiogenic potentials were determined using the western blot analysis, ELISA testing, scratch wound assay, transwell migration assay, and tube formation assay. To investigate the molecular mechanism, the lentivirus transfection was used. Ovariectomized and sham-operated rats with calvaria defect were analyzed using micro-CT, H\&E staining, Masson's trichrome staining, microfil perfusion, sequential fluorescent labeling, and immunohistochemistry assessment after administrated with/without catalpol.

Results: Our results manifested that catalpol enhanced BMSC osteoblastic differentiation and promoted BMSCmediated angiogenesis in vitro. More importantly, this was conducted via the JAK2/STAT3 pathway, as knockdown of STAT3 partially abolished beneficial effects in BMSCs. Besides, catalpol administration facilitated bone regeneration as well as vessel formation in an OVX-induced osteoporosis calvarial defect rat model.

Conclusions: The data above showed that catalpol could promote osteogenic ability of BMSC and BMSCdependent angiogenesis through activation of the JAK2/STAT3 axis, suggesting it may be an ideal therapeutic agent for clinical medication of osteoporotic bone fracture.

Keywords: Catalpol, Bone marrow mesenchymal stem cells, JAK2/STAT3 signaling, Osteoporosis, Bone repair

\footnotetext{
* Correspondence: longo472@outlook.com

'Department of Orthopaedic Surgery, The Second Affiliated Hospital and

Yuying Children's Hospital of Wenzhou Medical University, Wenzhou 325000,

China

${ }^{2}$ Key Laboratory of Orthopaedics of Zhejiang Province, Wenzhou 325000,

China

Full list of author information is available at the end of the article
}

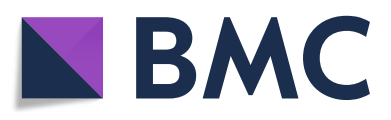

(- The Author(s). 2021 Open Access This article is licensed under a Creative Commons Attribution 4.0 International License, which permits use, sharing, adaptation, distribution and reproduction in any medium or format, as long as you give appropriate credit to the original author(s) and the source, provide a link to the Creative Commons licence, and indicate if changes were made. The images or other third party material in this article are included in the article's Creative Commons licence, unless indicated otherwise in a credit line to the material. If material is not included in the article's Creative Commons licence and your intended use is not permitted by statutory regulation or exceeds the permitted use, you will need to obtain permission directly from the copyright holder. To view a copy of this licence, visit http://creativecommons.org/licenses/by/4.0/. The Creative Commons Public Domain Dedication waiver (http://creativecommons.org/publicdomain/zero/1.0/) applies to the data made available in this article, unless otherwise stated in a credit line to the data. 


\section{Background}

Osteoporosis is becoming a worldwide health issue and a social threat due to the aging society. It is characterized by decreasing bone mass and deteriorating microarchitecture in the skeleton, both of which enhance the risk of bone fracture [1-3]. Even though the healing capacity of the bone tissue has been proved, a promising recovery of bone defect continues to be a challenging issue [4]. Various medical applications including autologous bone grafts and autografts have been used in the clinical options. However, these techniques encounter limitations because of the donor unavailability and morbidity, infection, immune rejection, pain, and the cost [5]. Herein, other therapeutic alternatives are necessary.

It is believed that bone formation, as well as fracture healing, is associated with moderate osteogenesis and angiogenesis [6]. Meanwhile, the communication between the BMSCs and vascular endothelial cells seems to be vital in the process of bone remodeling, which can be augmented by multiple regulatory factors that incorporate in the signaling modes of autocrine and/or paracrine, so as to participate in the recruitment, proliferation, and differentiation of BMSCs and vascular endothelial cells [7]. This connection has been demonstrated to have resulted from the osteoblastic and angiogenic factor production such as VEGF [8]. However, osteogenesis and angiogenesis under osteoporosis conditions are normally impaired due to the decreased recruitment of the osteoblastic cells to the damaged areas, consequently resulting in a nonunion of bone fracture [9-12]. Therefore, maintaining osteogenesis and angiogenesis is crucial for the osteoporotic bone regeneration.

Various signaling pathways are reported to be involved in the process of bone regeneration, among which Janus kinase/signal transducers and activators of transcription (JAK/STAT) pathways are indispensable. STAT3 is activated and phosphorylated by the JAK family particularly JAK2. This leads to STAT3 dimerization and nucleus translocation, and the initiation of the subsequent biological responses [13]. Previous studies have reported that the increase of the phosphorylation-STAT3 in BMSCs promotes osteogenic differentiation and enhances bone defect healing [14, 15]. Similarly, specific knockout of STAT3 potently reduces bone mineral density in mice [16]. In addition, JAK2/STAT3 axis inhibition contributes to the suppression of VEGF expression in bone marrow multipotent progenitor cells and BMSCs as well $[17,18]$. These provide evidence that osteogenesis and angiogenesis in bone healing are greatly associated with the activation of the JAK2/ STAT3 pathway.

Rehmanniae is a traditional Chinese herbal medicine that is used for the treatment of osteoporosis for hundreds of years [19]. However, its active part as well as the mechanism underlying its function has not been exhaustively explored. Catalpol is a natural iridoid glycoside that is dominantly enriched in the dried root of Rehmanniae, which possesses a diverse range of biological activities, such as anti-ischemic, anti-oxidative, and anti-inflammatory effects [20-22]. However, its potential actions on bone formation have not been fully elucidated. Recently, the association between the JAK2/ STAT3 pathway and the actions of catalpol has been previously reported [23].

On this basis, we hypothesized that catalpol could promote BMSC osteogenesis and BMSC-mediated angiogenesis by activating the JAK2/STAT3 axis. The current study was to lay the foundation for the application of catalpol in the treatment of the clinical osteoporotic bone fracture.

\section{Methods}

\section{Reagents and antibodies}

Catalpol (> 96\%) was purchased from Sigma-Aldrich (St. Louis, MO, USA). Primary antibodies against alpha-1 type I collagen (COL1A1), runt-related transcription factor 2 (RUNX2), and osteocalcin (OCN) were from Abcam (Cambridge, UK). The primary antibody against VEGF, CD31, p-JAK2, t-JAK2, p-STAT3, t-STAT3, and $\beta$-actin was obtained from the Cell Signaling Technology (Danver, MA, USA). Fetal bovine serum (FBS), minimum essential medium-alpha modification ( $\alpha$-MEM), and penicillin/streptomycin were purchased from Gibco BRL (Thermo Fisher Scientific; Waltham, MA, USA). All the other chemicals used were of analytical grade complying with the tissue and cell culture standards.

\section{Isolation and culture of rat BMSCs}

BMSC isolation was performed as previously described [24]. Briefly, 2-week-old Sprague-Dawley rats were euthanized and sterilized in $75 \%$ ethanol for $15 \mathrm{~min}$. BMSCs were flushed out by injection of modified Eagle's medium alpha modification ( $\alpha$-MEM; HyClone, PA, USA) using a 5 -ml syringe fitted with a 25 -gauge needle under sterile conditions. After centrifugation, the BMSCs were cultured in $\alpha$-MEM supplemented with $10 \%$ fetal bovine serum (FBS; Gibco, New York, USA) and $1 \%$ penicillin/streptomycin (Gibco) and incubated at $37{ }^{\circ} \mathrm{C}$ with $5 \% \mathrm{CO}_{2}$. The BMSCs from passages 3-5 were used in all experiments.

\section{Cytotoxicity assay}

The effect of catalpol on BMSC viability was determined by Cell Counting Kit-8 assay using commercial kits (MedChemExpress LLC; Monmouth Junction, NJ, The USA). Briefly, BMSCs were seeded in a 96-well plate at a density of $5 \times 10^{3}$ cells per well and various concentrations of catalpol were added $(0,10,25,50,100,250$, 
$500 \mu \mathrm{M})$ for 24 or $48 \mathrm{~h}$. At the end of the experiment, a $10-\mu$ CCK- 8 solution was added into the wells. The cells were cultured for 2 more hours. The optical density (OD) at the wavelength of $450 \mathrm{~nm}$ was measured via a Multiskan Go Microplate Spectrophotometer (Thermo Fisher Scientific).

\section{Osteoblastic determination and mineralization protocols} Initially, BMSCs were seeded at a density of $5 \times 10^{4}$ cells per well in a 24 -well plate. After reaching over $80 \%$ confluence, the medium was replaced with osteogenic medium (complete $\alpha$-MEM containing $1 \mathrm{nM}$ dexamethasone, $50 \mu \mathrm{M}$ ascorbic acid, and $20 \mathrm{mM} \quad \beta$ glycerophosphate). For treatment, catalpol (50, 100, and $250 \mu \mathrm{M})$ was added into the medium and the untreated wells were redeemed as the control group. Total cellular proteins were extracted after 7 days of osteogenic induction for western blot analysis of proteins involved in osteoblastic differentiation. Alkaline phosphatase (ALP) activity was detected on the 7th day of differentiation using the ALP Staining Kit (Beyotime Institute of Biotechnology; Jiangsu, China). The mineralization of calcium nodule was determined at the 21st day using Alizarin Red S (ARS) solution (Solarbio Science \& Technology). The absorbance at $520 \mathrm{~nm}$ for ALP and $570 \mathrm{~nm}$ for ARS staining was detected using a microplate reader.

\section{Western blotting}

The total cell protein was extracted from the cultured cells using RIPA lysis buffer (Beyotime Institute of Biotechnology), containing protease and phosphatase inhibitors (Sigma-Aldrich), for $30 \mathrm{~min}$ at $4{ }^{\circ} \mathrm{C}$. The cell lysates were cleared by centrifugation, and the protein concentration was determined using the BCA Protein Assay Kit (Beyotime Institute of Biotechnology) with respect to the manufacturer's instruction. For each sample, $20 \mu \mathrm{g}$ of the extracted protein (diluted in SDS Sampling buffer and denatured by boiling for $5 \mathrm{~min}$ ) were resolved on a 10-15\% SDS-polyacrylamide gel electrophoresis gel. The separated proteins were then transferred to polyvinylidene difluoride (PVDF) membranes (Merck Millipore; Burlington, MA, USA) overnight at $4{ }^{\circ} \mathrm{C}$. PVDF membranes were blocked with $5 \%$ skim milk diluted in Tris-buffered saline with a $0.1 \%$ Tween 20 (TBST) for 2 $\mathrm{h}$ at room temperature. They were then incubated with the primary antibodies for $12 \mathrm{~h}$ at $4{ }^{\circ} \mathrm{C}$. After extensive washes with TBST, the membranes were incubated with the corresponding HRP-conjugated secondary antibodies for $4 \mathrm{~h}$ at room temperature. The proteins were visualized through chemiluminescence and imaged on a ChemiDoc XRS+ (Bio-Rad; Hercules, CA, USA). The protein bands were quantified by densitometry analysis using Image Lab V3.0 software (Bio-Rad).

\section{Determination of angiogenesis-related functions of BMSCs}

To determine whether catalpol could enhance BMSCmediated angiogenic ability, the cells received $100 \mu \mathrm{M}$ catalpol treatment and they were cultured for $0,12,24$, or $48 \mathrm{~h}$. Besides, BMSCs were treated with various concentrations of catalpol $(0,50$, and $100 \mu \mathrm{M})$ for $24 \mathrm{~h}$. The protein was extracted and the supernatant liquid harvested at the end of the treatment, for western blotting analysis and ELISA test of VEGF. For further assessment of the angiogenic capability, the BMSCs were incubated with/without $100 \mu \mathrm{M}$ catalpol for $24 \mathrm{~h}$, and the conditioned mediums were harvested and centrifuged at $2000 \times g$ for $10 \mathrm{~min}$ to obtain the supernatants, which were used for downstream experiments. Subsequently, HUVECs were cultured under different treating conditions: (1) fresh medium, (2) fresh medium added with $100 \mu \mathrm{M}$ catalpol, (3) conditioned medium from BMSCs without catalpol, and (4) conditioned medium from BMSCs with $100 \mu \mathrm{M}$ catalpol.

\section{ELISA testing}

Commercial ELISA kit for VEGF (Cusabio, Wuhan, China) was used to determine the concentrations of VEGF in the culture medium after BMSC administration indicated the treatment, in accordance with the manufacturer's protocols.

\section{Migration assays}

Human umbilical vein endothelial cells (HUVECs) were purchased from Procell Life Science \&Technology Company (Wuhan, China). For the scratch wound assay, HUVECs were seeded in a 12-well plate at a density of $2.0 \times 10^{5} /$ well. After confluence, the cells were scratched using a sterile pipette tip under an inverted microscope (Nikon; Tokyo, Japan). The cells were then cultured in mediums aforementioned. The wound images were obtained immediately, 12 and $24 \mathrm{~h}$ later. The width of the wounded areas was calculated as previously described: width of the area $(\%)=\left(A_{0}-A_{n}\right) / A_{0} \times 100$, where $A_{0}$ represents the initial wound area, and $A_{n}$ represents the residual wound area at the metering point. For the transwell migration assay, HUVECs were suspended at a density of $1 \times 10^{4} /$ well and loaded into the top chamber of a 24-well, $8-\mu \mathrm{m}$ pore-size transwell plate (Corning, NY, USA). Then, the medium from the treated BMSCs was added to the below chamber. After $10 \mathrm{~h}$, the unmigrated HUVECs in the upper chambers were removed by wiping the top of the membranes with cuspidal cotton swabs. After fixing in a $4 \%$ paraformaldehyde and washing with PBS solution, the migrated cells which were stained with $0.5 \%$ crystal violet for $2 \mathrm{~h}$. They were imaged as well as counted under the random fields of the microscope (Nikon; Tokyo, Japan). 


\section{Tube formation assay}

HUVECs were seeded at the density of $1.5 \times 10^{4} /$ well onto Matrigel-coated 96-well plate and then incubated in the medium aforementioned. After $8 \mathrm{~h}$ of culture, the tube formation of HUVECs was observed under an inverted microscope (Nikon; Tokyo, Japan). The number of tubes was determined using the Image-Pro Plus software.

\section{Immunofluorescence}

After the treatment, the cells were fixed in a $4 \%$ paraformaldehyde for $10 \mathrm{~min}$ at room temperature and permeabilized with a $0.5 \%(\mathrm{v} / \mathrm{v})$ Triton X-100 in PBS for 20 min. Non-specific antibody binding was blocked with a $1 \%(\mathrm{w} / \mathrm{v})$ goat serum albumin for $1 \mathrm{~h}$ at room temperature. It was then incubated with indicated antibodies (the dilution for the antibodies was 1:200 respectively) in a $0.2 \%$ BSA-PBS overnight at $4{ }^{\circ} \mathrm{C}$, with gentle mechanical rocking. The cells were washed extensively and incubated with fluorescence conjugated secondary antibodies (Alexa Fluor 488 or 594; Thermo Fisher Scientific) for $1 \mathrm{~h}$ at room temperature, in the dark. The nuclei were stained with DAPI for $5 \mathrm{~min}$ at room temperature. Fluorescence images were captured on an Olympus BX53 fluorescence microscope (Olympus Life Science; Tokyo, Japan). The level of expression was determined using integrated optical density using the Image-Pro Plus software (Media Cybernetics, Inc.; Rockville, MD, USA).

\section{Lentivirus infection}

STAT3 was suppressed by STAT3 knockdown lentivirus transfection (GeneChem, Shanghai, China). The BMSCs received STAT3 knockdown lentivirus or vehicle lentivirus transfection. After 12-h incubation, the medium was replaced and the cells were cultured for 2 days to reach a $90-95 \%$ confluence. The transfected cells were used in the subsequent experiments. The successfully transfected cells were observed under a fluorescence microscope (Olympus Life Science; Tokyo, Japan) whereas the untreated cells were invisible. The impact of STAT-3 knockdown transfection was detected via western blot analysis.

\section{Surgery and treatment}

All the animal experiments were approved by the Animal Ethics Committee of The Second Affiliated Hospital and the Yuying Children's Hospital of Wenzhou Medical University. They were carried out with respect to the criteria outlined in the Guide for the Care and Use of Laboratory Animals (NIH, Bethesda, MD, USA). Thirty 3month-old SD female rats were purchased from the Shanghai Laboratory Animal Center (SLACCAS; Shanghai, China). They were housed in ventilated cages in groups of 5 under the SPF conditions of $22-25^{\circ} \mathrm{C}$ and 12-h daylight. Twenty of the 30 rats were subjected to bilateral ovariectomy, and the rest underwent the sham operation and regarded as the SHAM group. After 3 months, the rats were screened to prove osteoporotic bone loss through the X-ray radiograph imaging. Then, all the rats were anesthetized, and 4-mm calvarial bone defects were made to determine osteogenesis and angiogenesis function as described in our previous study [25, 26]. After surgery, all the ovariectomized (OVX) rats were divided into 2 groups: OVX and OVX + catalpol. The rats in the OVX + catalpol group received $10 \mathrm{mg} /$ $\mathrm{kg} /$ day catalpol intraperitoneal injection while those in the other groups were subjected to vehicle (PBS) treatment. The treatments were performed daily for 8 weeks. At the end of the treatment, all rats were euthanatized and the calvarial bones were harvested and fixed in a $4 \%$ formaldehyde.

\section{Micro-CT}

Microstructural analysis of the calvaria of the rats was scanned on a micro-CT system and the associated software $(\mu \mathrm{CT} 100$, Scanco Medical; Brüttisellen, Switzerland). The images were acquired at a voltage of $70 \mathrm{kV}$, an electric current of $200 \mu \mathrm{A}$, and a spatial resolution of $14.8 \mathrm{~mm}$ in all the directions. Threedimensional reconstructed images were generated. The volume of interest (VOI) analyzed included the trabecular compartment $2 \mathrm{~mm}$ below the highest point of the growth plate to distal $100 \mathrm{CT}$ slices. The volume of the regenerated bone within the calvaria defects, and bone formation was analyzed using the built-in software.

\section{Microfil perfusion}

Calvarial blood vessel formation in the defect area was evaluated by perfusion with Microfil compound (Microfifil MV-122; Flow Tech) 8 weeks after craniotomy surgery. The rat was initially scraped clean and immobilized on a foam board following anesthesia. An incision was created between the xiphoid and the abdomen. The inferior vena cava was cut and flushed with PBS containing sodium heparin $(100 \mathrm{U} / \mathrm{ml})$. The vessel was then fixed via injection of $4 \%$ formaldehyde. Then, $20 \mathrm{ml}$ of silicone rubber compound was injected into the vessel at a rate of $2 \mathrm{ml} / \mathrm{min}$. Later, the specimen was stored overnight at $4{ }^{\circ} \mathrm{C}$, and fixed in a $4 \%$ paraformaldehyde for a further $48 \mathrm{~h}$. The tissues were then after decalcified in $10 \%$ ethylene diamine tetra acetic acid (EDTA; SigmaAldrich, MO) for 4 weeks. Finally, the vessel formation of the cranial defect was analyzed using a micro-CT system.

\section{Sequential fluorescent labeling}

Continuous fluorescent labeling for the detection of bone mineralization in calvaria defects was carried out. 
Rats were injected with calcein $(15 \mathrm{mg} / \mathrm{kg}$ of body weight, CA Sigma USA) intraperitoneally at 4 and 6 weeks after the surgery. Fluorescent agents were prepared instantly before injection and filtered using a $0.45-$ $\mu \mathrm{m}$ filter. The calvaria tissue samples were harvested for hard-tissue slicing and imaged through the fluorescence microscopy (Olympus Life Science; Tokyo, Japan).

\section{Histology and immunohistochemistry assessment}

Fixed calvaria bone tissues were decalcified in 10\% EDTA for 3 weeks for histological and immunohistochemical (IHC) analyses. Then, the specimen was cut in half, dehydrated in graded ethanol series (70\% to 100\%), cleared in xylene, and paraffin-embedded for sectioning. Four-micrometer-thick sections were then subjected to

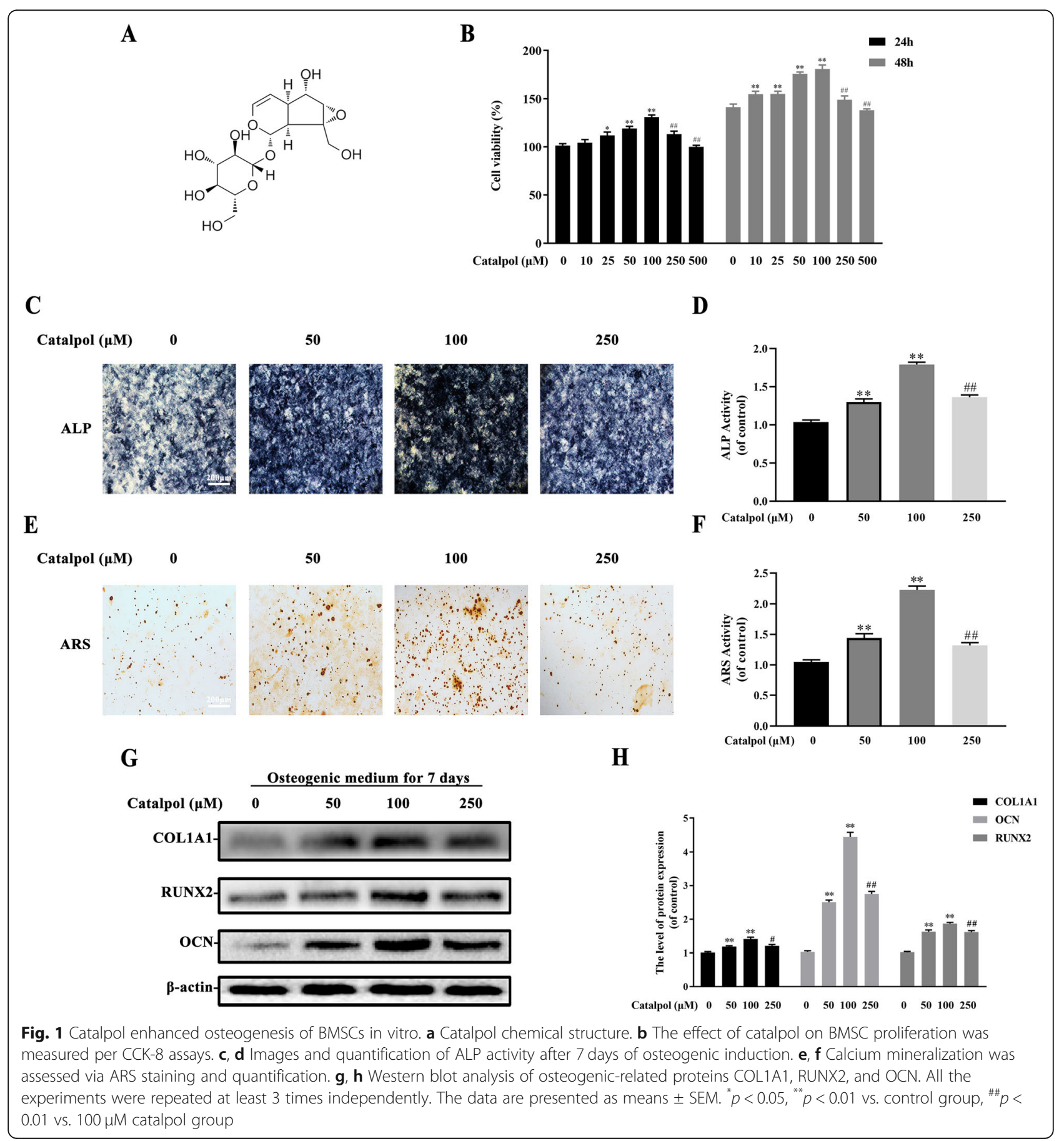


A

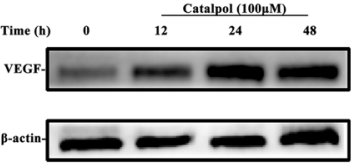

D

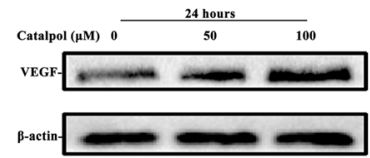

B

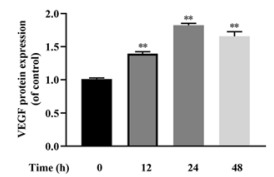

E

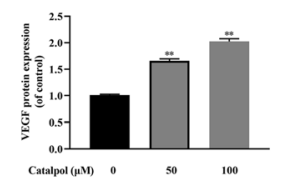

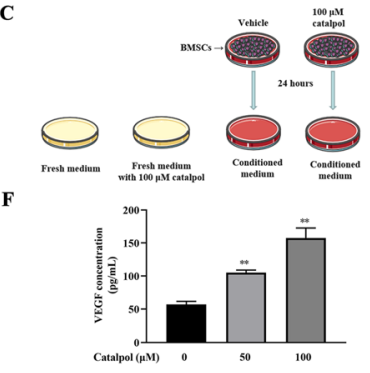

G

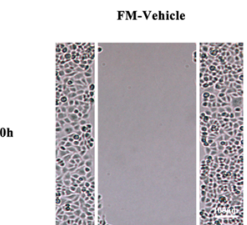

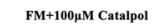
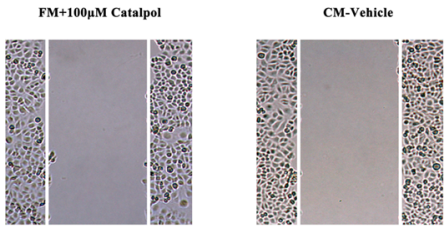

$\mathrm{CM}+100 \mu \mathrm{M}$ Catalpol

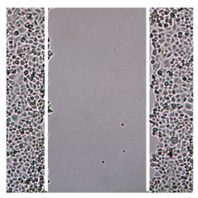

${ }^{12 \mathrm{~h}}$
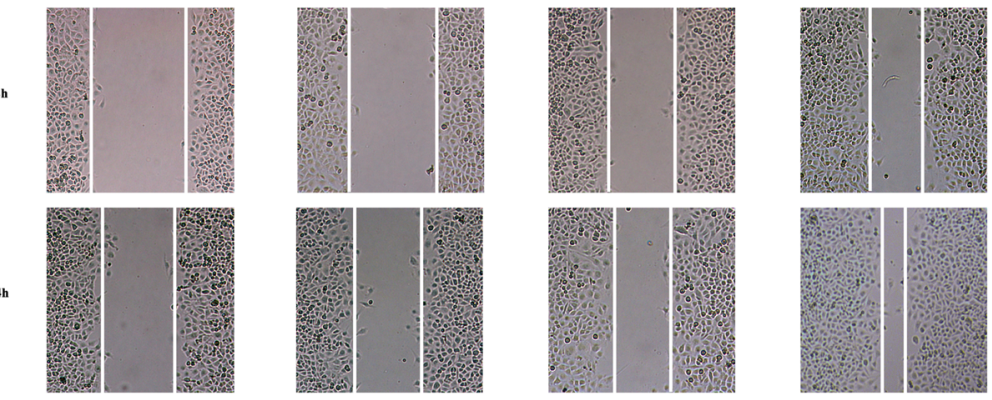

H
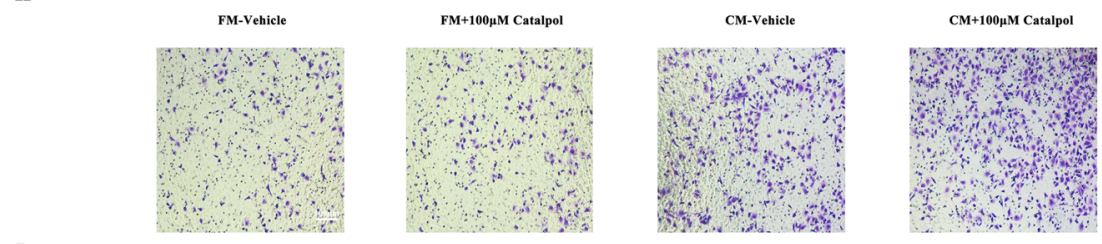

FM-Vehicle

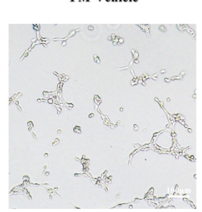

FM+100 MM Catalpol

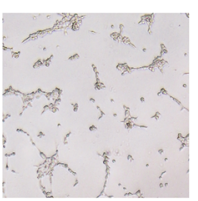

CM-Vehicle

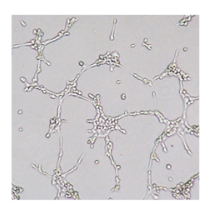

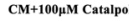

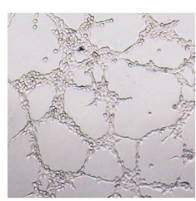

$\mathbf{K}$

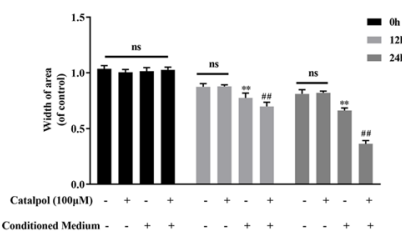

$=$
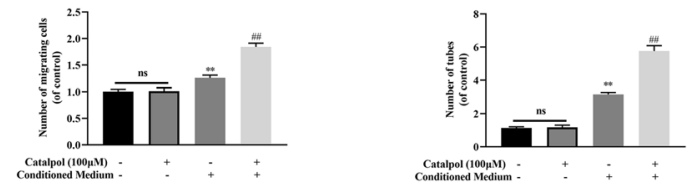

Fig. 2 Catalpol augmented BMSC-dependent angiogenesis of HUVECs. a, b Western blot analysis of VEGF expression in BMSCs after indicated treatment. c Diagram of groups dividing and treatment respectively, $\mathbf{d}$, e Western blot analysis of VEGF expression in BMSCS after indicated treatment. $\mathbf{f}$ VEGF secretion in the supernatant liquid from different groups was assessed using ELISA kits. Images and quantification of $\mathbf{g}$, $\mathbf{j}$ scratch wound assay, $\mathbf{h}, \mathbf{k}$ transwell migration assay, and $\mathbf{i}$, I tube formation assay of HUVECs incubated with indicated mediums. The data are presented as means \pm SEM. All experiments were repeated at least 3 times independently. ${ }^{* *} p<0.01$ vs. control group, ${ }^{\# \#} p<0.01$ vs. CM-vehicle group. (FM fresh medium, CM conditioned medium) 
hematoxylin and eosin (H\&E), and Masson's trichrome staining as per the standard laboratory protocols. For IHC staining, 6- $\mu \mathrm{m}$ sections were incubated with primary antibodies against CD31, VEGF, and OCN. The immune-reactivities of the sections were determined using horseradish peroxidase detection system in accordance with manufacturer's protocols (Vector Laboratories; Burlingame, CA, USA).

\section{Statistical analyses}

All statistical analyses were carried out using the GraphPad Prism software (San Diego, CA, USA). The data presented herein are expressed as the mean \pm standard error of the mean from at least three experimental repeats. Two-tailed Student's $t$ test was used to compare the means between two groups and one-way ANOVA with Bonferroni or Dunnett corrections were used for multiple comparisons where appropriate. Differences were determined to be statistically significant when $p<0.05$.

\section{Results}

Catalpol enhanced osteogenesis of BMSCs in vitro

Catalpol is a natural iridoid glycoside. It is a monoterpene containing a glucose molecule (Fig. 1a). To determine whether catalpol affects the proliferation of BMSCs, we initially performed CCK- 8 assays. Cell proliferation was not significantly suppressed by treatment with 10,25 , and $50,100 \mu \mathrm{M}$ catalpol for $24 \mathrm{~h}$ or $48 \mathrm{~h}$ compared to the control group (Fig. 1b). However, there was no more favorable change in cell viability when incubated with higher concentrations of catalpol $(250,500 \mu \mathrm{M}, P<0.05)$. Then, we assessed the pro-osteogenic effect of catalpol on BMSCs in vitro. After 7 days of osteoblastic induction, BMSCs treated with 50 or $100 \mu \mathrm{M}$ catalpol indicated an increasing level of ALP activities (Fig. 1c, d), in comparison to the control $(P<0.05)$. Consisting with the result of CCK- 8 assay, the rewarding effect was partly attenuated in BMSCs when incubated with a higher concentration of catalpol

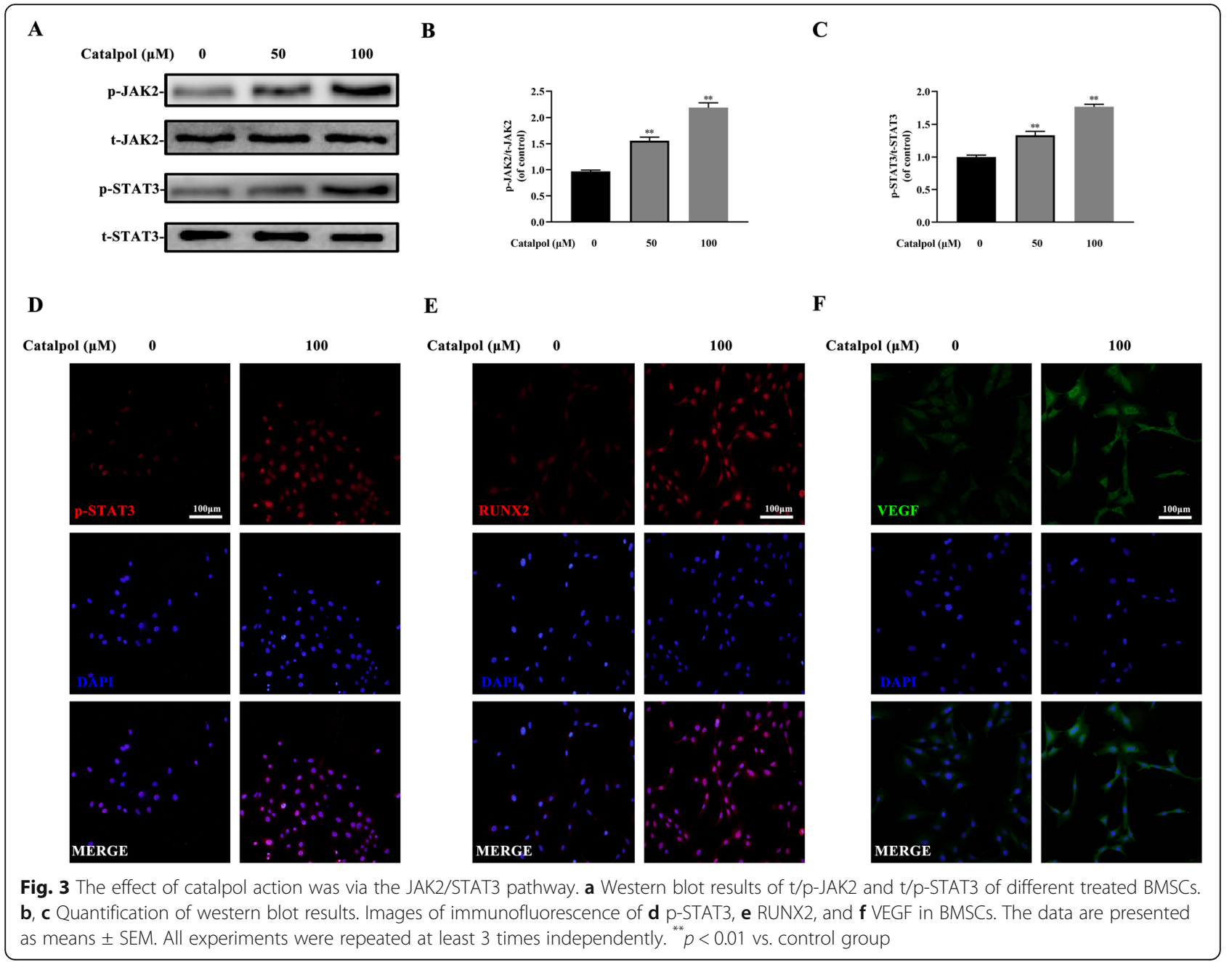


$(250 \mu \mathrm{M})$. It was also validated by ARS staining, which epresented extracellular deposition of calcium after 21 days of osteogenic induction (Fig. 1e, f). Accordingly, the results of the western blot analysis of osteogenesis-related proteins COL1A1, RUNX2, and OCN also revealed that catalpol promoted osteoblastic gene expression compared to the control $(P<0.05)$ (Fig. 1g, h).
Catalpol augmented BMSC-dependent angiogenesis of HUVECs

Since VEGF is a critical pro-angiogenesis factor in the crosstalk between BMSCs and endothelial cells, we first evaluated the impact of catalpol on BMSCs VEGF expression and secretion. It was realized that VEGF expression was highest $24 \mathrm{~h}$ after catalpol application (Fig. 2a, b). We also observed that VEGF expression and

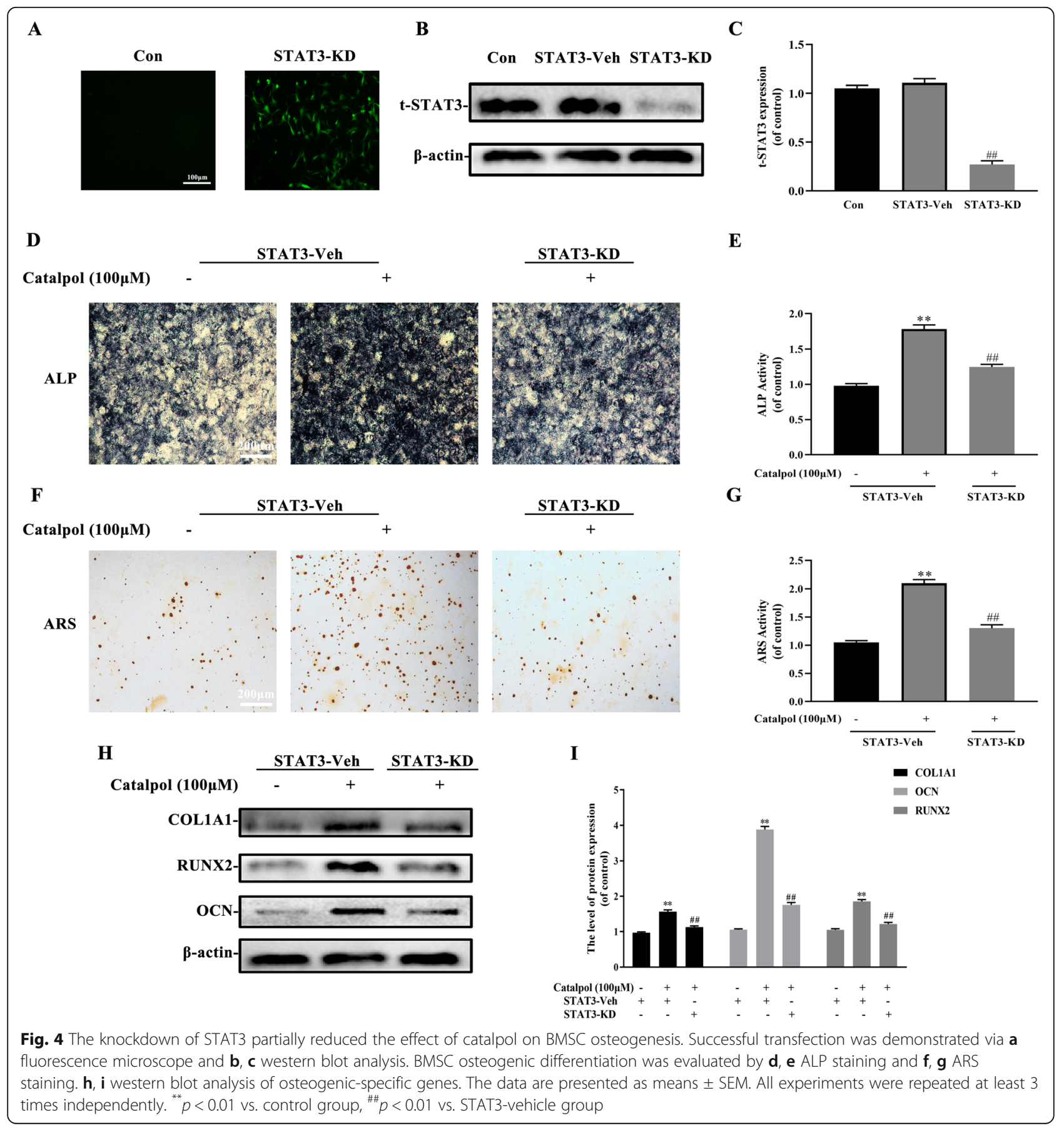


secretion were enhanced in a dose-dependent manner (Fig. 2d-f). To further explore the catalpol effect on BMSC-dependent angiogenesis, we treated BMSCs with/ without $100 \mu \mathrm{M}$ catalpol for $24 \mathrm{~h}$ then collected the respective conditioned mediums to find out whether catalpol treatment could facilitate BMSC-induced proangiogenic impacts of HUVECs (Fig. 2c). As evidenced by the scratch wound assay and transwell migration assay, the conditioned medium from catalpol treated BMSCs resulted in an incredible increase in HUVEC migration in comparison to the untreated group (Fig. $2 \mathrm{~g}$, h, $\mathrm{j}, \mathrm{k}, P<0.05)$. Also, catalpol treatment enhanced BMSCrelated capillary tube-like structures on Matrigel (Fig. 2i, 1). Besides, our results demonstrated that catalpol treatment was not able to facilitate HUVEC angiogenesis. These findings suggested that catalpol possessed the ability to promote BMSC-mediated angiogenesis by promoting VEGF expression.

\section{JAK2/STAT3 axis activation contributed to the impacts of catalpol on osteogenesis-angiogenesis coupling}

To determine the role of JAK2/STAT3 pathway in the effect of catalpol, BMSCs were treated with 50 and $100 \mu \mathrm{M}$ catalpol for $24 \mathrm{~h}$. The western blot analysis result demonstrated that catalpol increased phosphorylation of JAK2 and STAT3 (Fig. 3a-c). Treatment of $100-\mu \mathrm{M}$ catalpol significantly increased nucleus p-STAT3 translocation and the expression of RUNX2 and VEGF as proved by immunofluorescence (Fig. 3d-f). To further explore the role of STAT3 on the effect of catalpol, we suppressed STAT3 expression via lentivirus transfection. A successful

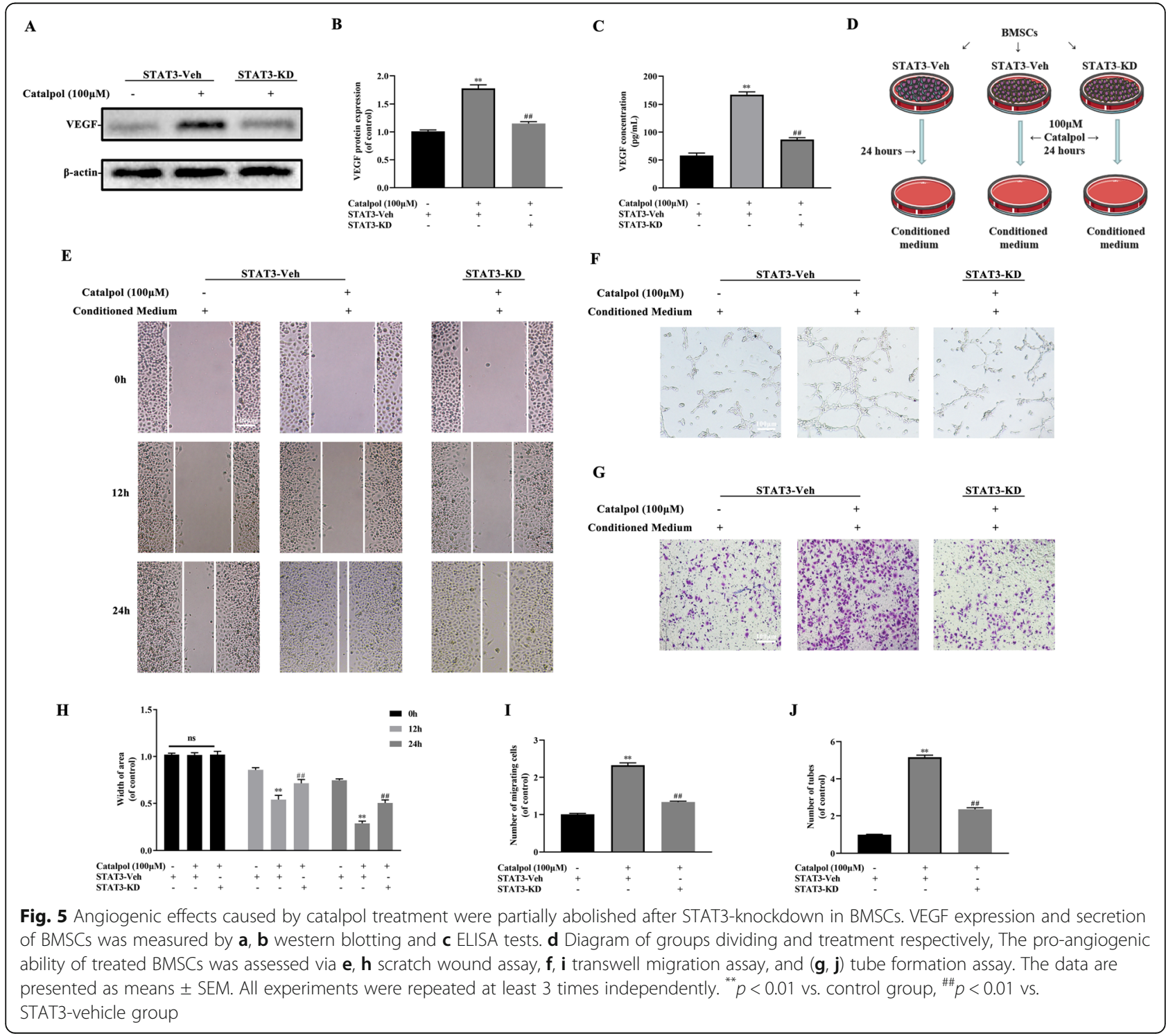


transfection was carried out as the transfected BMSCs were fluorescent while the untreated cells were invisible under a fluorescent microscope (Fig. 4a). BMSCs treated with STAT3 knockdown lentivirus showed a lower level of STAT3 expression as compared to the others $(P<0.05)$ (Fig. $4 \mathrm{~b}, \mathrm{c})$. We next determined if STAT3 was responsible for the effects of catalpol on BMSCs and realized that the knockdown of STAT3 diminished ALP activity and mineralization of BMSCs compared to the vehicle group (Fig. $4 \mathrm{~d}-\mathrm{g}$ ). Parallel results showed that osteogenesis-related proteins RUNX2, COL1A1, and OCN were downregulated in STAT3 knockdown BMSCs (Fig. 4h, i). As shown in
Fig. 5d, we next determined whether STAT3 was also involved in the pro-angiogenic effect of catalpol action. Our data showed that VEGF expression and secretion in catalpol-treated BMSCs were reduced after the STAT3 knockdown (Fig. 5a-c). A concomitant reduction in BMSC-induced pro-angiogenesis effect in the scratch wound assay, transwell migration assay, and tube formation assay was also observed (Fig. 5ej). It was clear that this effect was attenuated after STAT3 blocking $(P<0.05)$. All these data demonstrated that the JAK2/STAT3 pathway was involved in the catalpol action on osteogenesis-angiogenesis coupling in vitro.
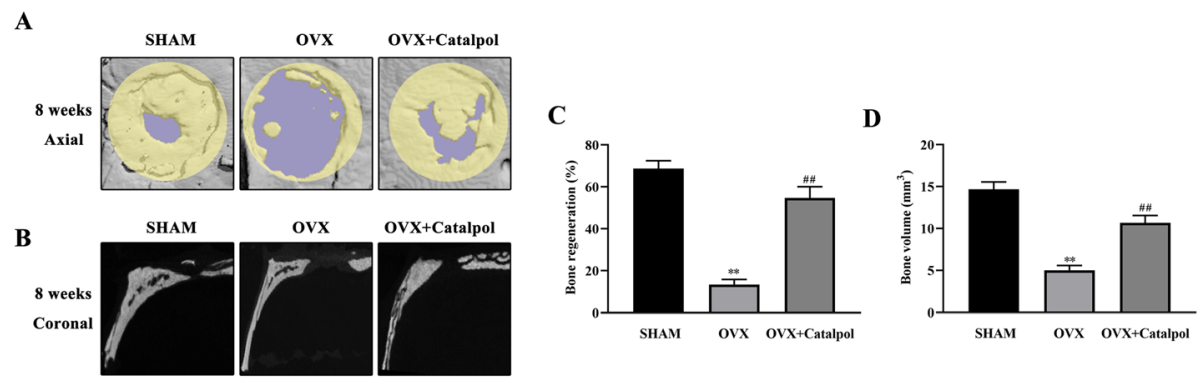

$\mathbf{E}$

SHAM

ovx

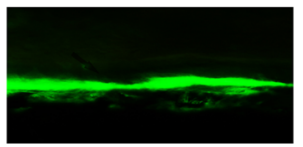

ovX+Catalpol

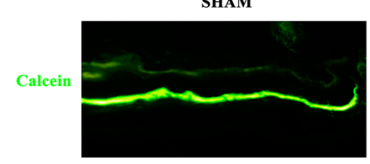

SHAM

$\mathbf{F}$

ovx
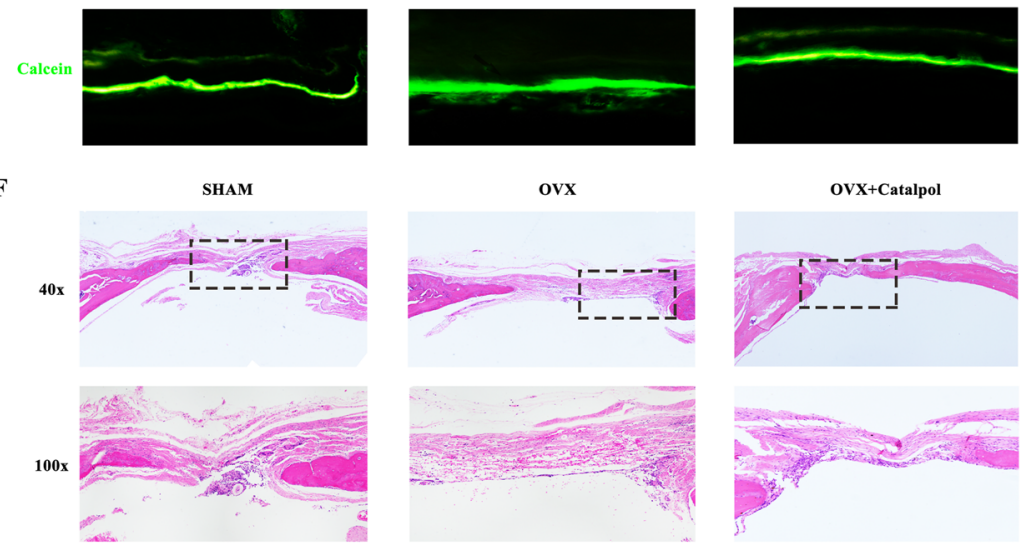

OvX+Catalpol

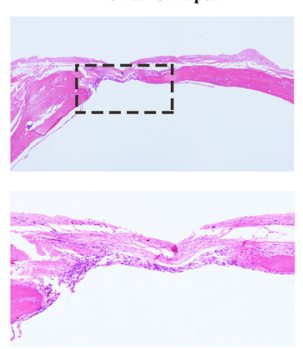

G

SHAM

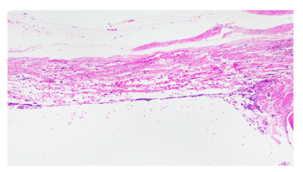

ovx

OvX+Catalpol
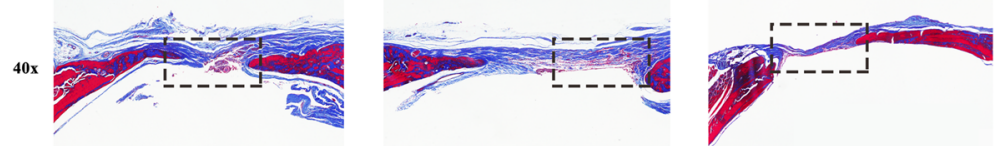

$100 \mathrm{x}$
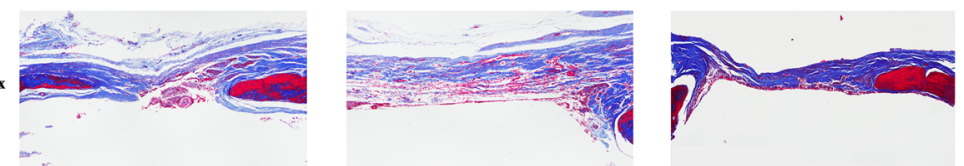

Fig. 6 Intraperitoneal administration of catalpol increased bone healing capacity in OVX calvarial defect rats. a-d Micro-CT images and analysis of calvaria defect of different groups of rats. e New bone formation was detected by sequential fluorescent labeling of calcein. Histological assessment of the defect area by $\mathbf{f}$ H\&E staining and $\mathbf{g}$ Masson trichrome staining. The data are presented as means \pm SEM. ${ }^{* *} p<0.01$ vs. SHAM group, ${ }^{\# \#} p<0.01$ vs. OVX group 
Intraperitoneal administration of catalpol increased bone healing capacity in OVX calvarial defect rats

We explored if the effects of catalpol on BMSCs and HUVECs could be recapitulated in vivo. Rats were first ovariectomized and then subjected to craniotomy and received catalpol/vehicle treatment for 8 weeks. The 3D reconstruction images of calvaria showed that an increase in bone regeneration area in the OVX + catalpol group as compared to the OVX group (Fig. 6a-d). This confirmed that catalpol treatment promoted bone formation in OVX rats. Accordingly, bone mineralization labeled by calcein at 4 weeks and 6 weeks after craniotomy revealed that the distance strip in the OVX + catalpol group which marked by green was wider than in the OVX group (Fig. 6e). Meanwhile, the H\&E staining and Masson trichrome staining of the calvarial bone showed that catalpol administration increased bone mineralization and formation around the defect than the OVX group (Fig. 6f, g).

\section{Catalpol treatment facilitated vessel formation in the defect area}

To observe vascular formation in calvaria defect, the rats were perfused with Microfil. A sparse vessel network was found in the OVX group while more vascular formation was detected in the OVX + catalpol group (Fig. 7a). With regard to the quantitative analysis, both the vessel area and vascular number increased when treated with catalpol, concomitant with the decrease in vessel separation as compared to the OVX group (Fig. $7 \mathrm{~b}-\mathrm{d})$. The results implied that catalpol treatment improved vessel formation in osteoporosis calvaria defect.

\section{Catalpol enhanced osteogenesis and angiogenesis in the calvarial defect in OVX rats}

To evaluate osteogenesis and angiogenesis-related gene expressions, we extracted the proteins from the bone tissue surrounded the bone defect. Western blot results manifested that RUNX2, OCN, and VEGF expressions were upregulated in the OVX + catalpol group as compared to the OVX group (Fig. 8a-d). Besides, the immunohistochemical staining and quantitative analysis of OCN, VEGF, and vessel marker CD31 revealed a more positive area in the OVX + catalpol group (Fig. $8 \mathrm{e}-\mathrm{h}$ ). Generally, these findings revealed that catalpol treatment improved bone formation and neovascularization in OVX rats.

\section{Discussion}

Bone defect repairing is a recapitulation process with complex signaling cascades, including spatiotemporal osteogenesis and angiogenesis, both of which require BMSC migration and osteogenic differentiation, accompanied by a well-organized blood vessel production [27,

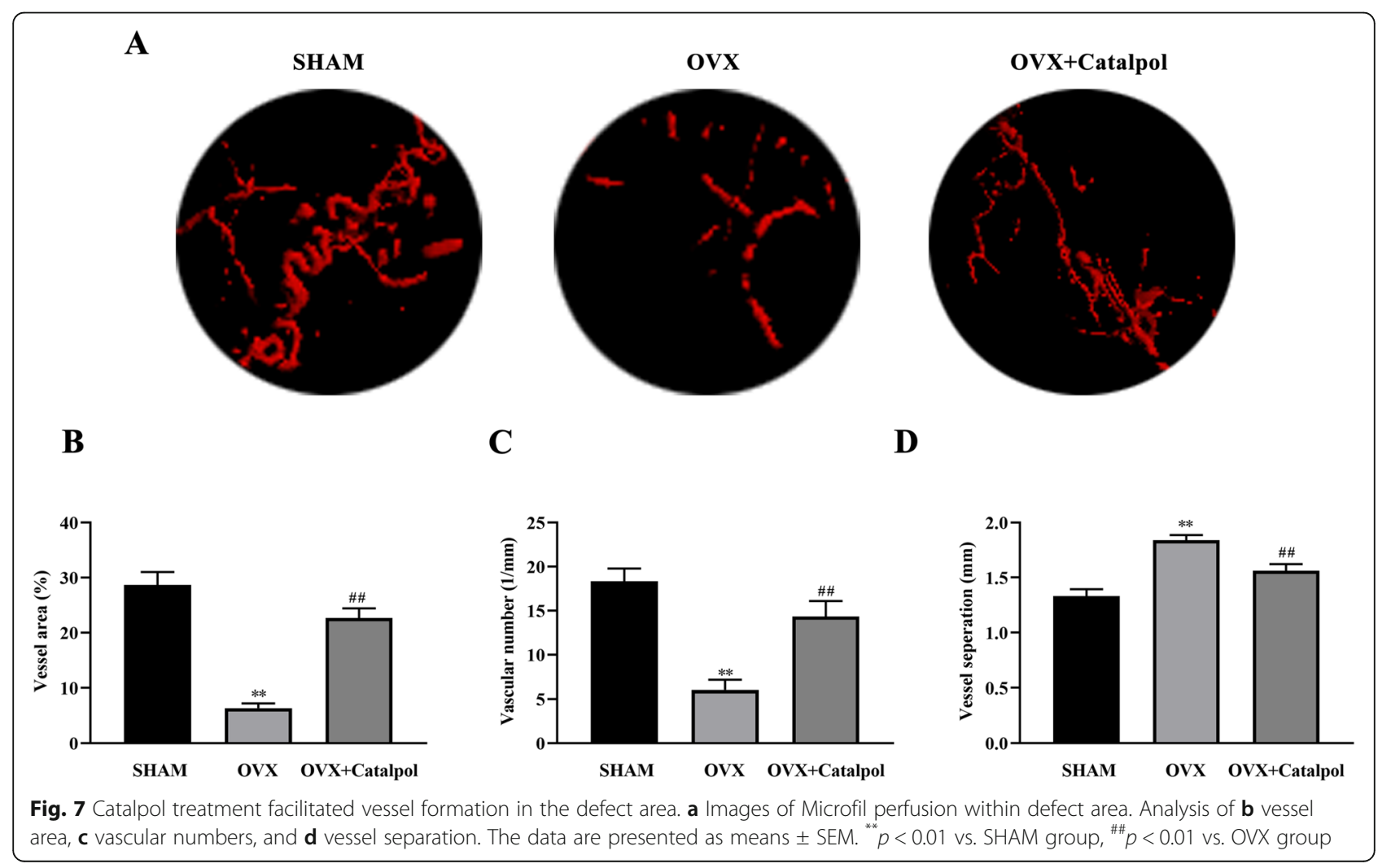




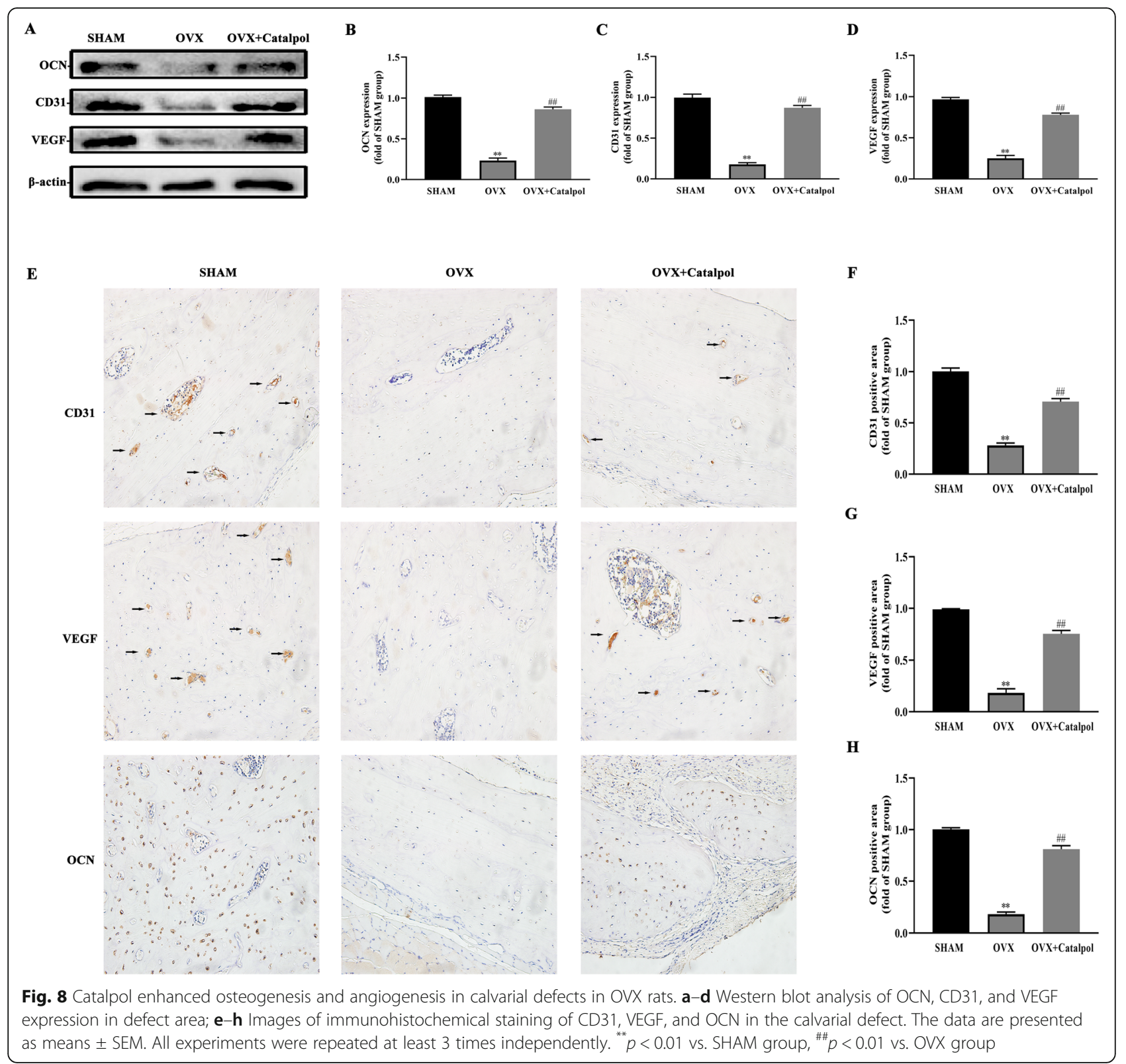

28]. However, the delayed union or even nonunion of bone fracture often occurs in osteoporosis patients due to the decreased osteogenesis and disordered angiogenesis [29]. Therefore, exploring more therapeutic strategies and ideal drugs for the treatment of osteoporosis fracture is vital for clinical application. To the best of our knowledge, the current study is the first to demonstrate that catalpol treatment could enhance BMSC osteogenic determination and BMSC-mediated angiogenesis through the JAK2/STAT3 pathway activation. Further, knockdown of STAT3 partially abolished the beneficial effects of catalpol on BMSCs in vitro. Meanwhile, in vivo experiments confirmed that catalpol administration accelerated bone repair as well as promoted angiogenesis in an OVX rat calvaria defect model. The mechanism of catalpol actions on BMSCs was as displayed in Fig. 9.

BMSCs are identified as multipotent, undifferentiated cells with regenerative ability. The BMSCs play a central role in postnatal bone regeneration, which comprises two main ossification processes, namely, endochondral ossification and intramembranous ossification [30]. When a fracture happens, BMSCs spontaneously migrate to the bone lesion and directly differentiate to osteoblasts, which secrete extracellular matrix to help in rapid bone repair [27]. The improvement of BMSC osteogenic lineage function is essential for the successful fracture healing especially the nonunion. To this end, it is 


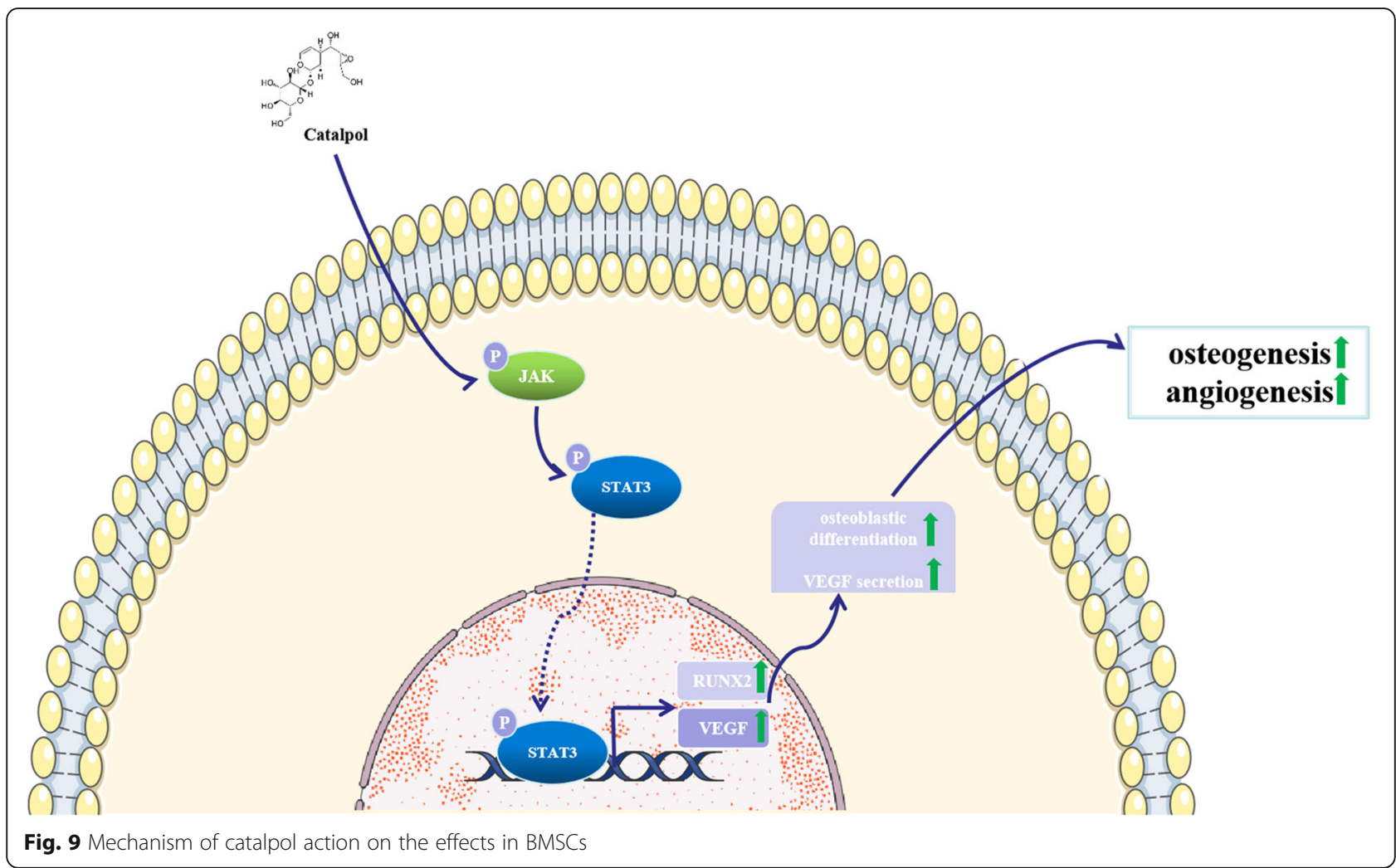

valuable to identify methods that could promote osteoblast differentiation of BMSCs for traditional medicine. To date, natural medicine has captured a lot of attention for its fascinating effects on promoting bone recovery. The study from Zhang et al. suggests that psoralen induced osteoblast determination and accelerated tibial fracture healing by activating ERK signaling [31]. Karvande et al. have reported the application of Heartwood extract from Dalbergia sissoo stimulates osteoblast function and promotes osteoporotic bone repair [32]. Rehmanniae, a traditional and official medicine which is listed in the Chinese Pharmacopeia, has been transformed into various therapeutic applications for multipurpose clinical uses such as osteoporosis treatment [19, 33]. Recently, a growing number of researchers have reported that catalpol, the main component of Rehmanniae, possesses favorable pharmacological properties on bone metabolism. A study by Zhu et al. on the effect of catalpol on rat BMSCs showed that catalpol treatment could promote BMSC osteogenesis and prevent OVXinduced bone loss [21]. According to their results, the pro-osteogenic effect of catalpol was involved with activation of the $\mathrm{Wnt} / \beta$-catenin pathway, as co-culture with Dickkopf-related protein 1 (DKK-1) partly inhibited the increased osteogenesis of BMSCs by treatment of catalpol. Their work was further validated by the study from Cheng et al., which demonstrated that catalpol could enhance the activity of Wnt/ $\beta$-catenin axis in MC3T3-E1 osteoblasts under high glucose condition [34]. Choi et al. also reported that catalpol prevents MC3T3-E1 osteoblasts apoptosis caused by TCDD-induced damage [35]. Lai and his colleagues found that catalpol could alleviate bone loss by mediating the Th1/Th2 cells paradigm [36]. Consistent with the previous findings, we showed that catalpol treatment enhanced ALP activity of BMSCs and augmented calcium mineralization in vitro, accompanied with elevated levels of osteogenic gene expression. In vivo results also revealed that catalpol application contributed to bone defect repair in OVX rats, confirming that catalpol treatment was potentially helpful to bone tissue regeneration.

The coordinated coupling of osteogenesis and angiogenesis is vital in the maintenance of the bone mass and bone regeneration, as blood vessels carry oxygen, materials, and nutrients that are critical to the bone development [30]. The impairment of neovascularization could inhibit trabecular bone regeneration and retard fracture healing. For example, bone density and strength considerably decline after surgical damage to the blood vessels $[9,37]$. Vascular endothelial growth factor (VEGF) has been regarded as one of the most critical factors for bone regeneration [38]. More importantly, VEGF from early osteoprogenitors is dispensable for periosteal angiogenesis and bone regeneration [12]. Our previous study also agreed with the perspective that vascular formation was indispensable in the bone repairing process 
$[39,40]$. In this current study, our data proved that catalpol administration promoted vessel formation in the calvaria defect area of OVX rats. Besides, VEGF secreted from catalpol-treated BMSCs could induce a higher level of HUVEC angiogenesis in vitro, which is consistent with Ju et al.'s discovery.

Previous studies have depicted the roles of the JAK2/ STAT3 signaling pathway in bone metabolism including osteogenesis and neovascularization [41]. The application of JAK2 inhibitor TG101348 as well as AG490 suppresses BMSC osteogenic differentiation and consequently impairs bone healing [42, 43]. Besides, STAT3 is the most influential transcription factor among the STATs family in mediating cellular signaling in bone-associated cells [44]. STAT3 regulates osteogenesis and angiogenesis through gene transcription by binding the specific gene-promoter sequences in the nucleus such as RUNX2 and VEGF [45, 46]. In humans, STAT3 mutation declines the bone mass and increases the incidence of trauma fractures [47]. This is normally the consequence of increased bone resorption and decreased bone formation. Besides, more evidence suggested that bone-selective STAT3 knockout mice indicated an osteoporotic phenotype [48, 49]. With regard to its role in vessel formation, the association between STAT3 and neovascularization has been widely elucidated. The activation of STAT3 promotes the angiogenic capability of endothelial cells both in vivo and in vitro [50]. Also, BMSC-secreted VEGF considerably decreased after the inhibition STAT3, resulting in a nonunion of the bone fracture $[14,18]$. Work from Dong et al. demonstrated that the effect of catalpol was mediated by the JAK2/STAT3 pathway [23]. In this study, we observed that co-culture with catalpol triggered JAK2 as well as STAT3 phosphorylation and promoted STAT3 nucleus-translocation in the BMSCs. Results from our study also indicated that BMSC osteogenic determination and BMSC-secreted VEGF, as well as its concomitant angiogenic effects, were partly abrogated after STAT3 knockdown. Additionally, we found this catalpol effect on BMSC-dependent VEGF secretion was consistent with previous study from Ju and his colleagues [51]. In general, these results indicated that catalpol exerted a coupling effect between osteogenesis and angiogenesis in BMSCs through JAK2/STAT3 activation.

\section{Conclusions}

Our findings highlight the beneficial effects of catalpol in promoting osteogenic and pro-angiogenic abilities of BMSCs. These impacts are preliminary considered via JAK2/STAT3 pathway activation. The application of catalpol may provide new insight and strategy for bone regeneration and hence could be an ideal therapeutic remedy against osteoporosis fracture.

\section{Abbreviations}

ALP: Alkaline phosphatase; ARS: Alizarin red S; BMSCs: Bone marrow mesenchymal stem cells; BCA: Bicinchoninic acid; BSA: Bovine serum albumin; COL1A1: Alpha-1 type I collagen; CCK-8: Cell counting Kit-8; DAPI: 4',6-Diamidino-2-phenylindole; DKK-1: Dickkopf-related protein 1; ELISA: Enzyme-linked immunosorbent assay; EDTA: Ethylene diamine tetraacetic acid; FBS: Fetal bovine serum; H\&E: Hematoxylin and eosin; HUVECs: Human umbilical vein endothelial cells; IHC: Immunohistochemical; JAKVSTAT: Janus kinase/signal transducers and activators of transcription; OCN: Osteocalcin; PVDF: Polyvinylidene fluoride; PBS: Phosphate buffer saline; RUNX2: Runt-related transcription factor 2; SEM: Standard error of mean; TCDD: Tetrachlorodibenzo-p-dioxin; VOI: Volume of interest; a-MEM: Modified Eagle's medium alpha modification

\section{Acknowledgements}

Not applicable.

\section{Authors' contributions}

All the listed authors made substantial contributions to the study. Liang Chen, Ri-Yan Zhang, Jun Xie, and Jia-Yi Yang participated in the experimental design and contributed the reagents, materials, and analytical tools. Lei Yang and Liang Chen wrote the manuscript. Liang Chen, Najeeb Bsoul, Chen-Xuan Hong, Rong-Bo Yang, and Kang-Hao Fang are involved in data analysis. All the data shown in the figure are from the above authors' experiments and can be used. The authors read and approved the final manuscript.

\section{Funding}

This research work was funded by research grants from the National Natural Science Foundation of China (Grant No.81772348), the National Key Research and Development Project of China (Grant No.: 2017YFB1304200), and the Wenzhou Science and Technology Bureau (Grant No.: Y20180028).

Availability of data and materials

All data generated or analyzed during this study are included in this published article.

Ethics approval and consent to participate

Not applicable

\section{Consent for publication}

Not applicable

\section{Competing interests}

The authors declare that they have no competing interests.

\section{Author details}

${ }^{1}$ Department of Orthopaedic Surgery, The Second Affiliated Hospital and Yuying Children's Hospital of Wenzhou Medical University, Wenzhou 325000, China. ${ }^{2}$ Key Laboratory of Orthopaedics of Zhejiang Province, Wenzhou 325000, China. ${ }^{3}$ School of Ophthalmology and Optometry, Wenzhou Medical University, Wenzhou 325000, China. ${ }^{4}$ Department of Gynecology, The First Affiliated Hospital of Wenzhou Medical University, Wenzhou 325027, China. ${ }^{5}$ Medical College, Zhejiang Jiaxing College, Jiaxing 314000, China.

Received: 28 October 2020 Accepted: 20 January 2021

Published online: 04 February 2021

\section{References}

1. Chalitsios CV, McKeever TM, Shaw DE. Incidence of osteoporosis and fragility fractures in asthma: a UK population-based matched cohort study. Eur Respir J. 2021;57(1):2001251. https://doi.org/10.1183/13993003.012512020

2. Raisz LG. Pathogenesis of osteoporosis: concepts, conflicts, and prospects. J Clin Invest. 2005;115:3318-25.

3. Manolagas SC. From estrogen-centric to aging and oxidative stress: a revised perspective of the pathogenesis of osteoporosis. Endocr Rev. 2010; $31: 266-300$

4. Kumar S, Wan C, Ramaswamy G, Clemens TL, Ponnazhagan S. Mesenchymal stem cells expressing osteogenic and angiogenic factors synergistically enhance bone formation in a mouse model of segmental bone defect. Mol Ther. 2010;18:1026-34. 
5. Wang W, Yeung KWK. Bone grafts and biomaterials substitutes for bone defect repair: a review. Bioactive Mater. 2017;2:224-47.

6. Grellier M, Granja PL, Fricain JC, Bidarra SJ, Renard M, Bareille R, Bourget C, Amédée J, Barbosa MA. The effect of the co-immobilization of human osteoprogenitors and endothelial cells within alginate microspheres on mineralization in a bone defect. Biomaterials. 2009:30:3271-8.

7. Clarkin CE, Emery RJ, Pitsillides AA, Wheeler-Jones CP. Evaluation of VEGFmediated signaling in primary human cells reveals a paracrine action for VEGF in osteoblast-mediated crosstalk to endothelial cells. J Cell Physiol. 2008:214:537-44.

8. Leach JK, Kaigler D, Wang Z, Krebsbach PH, Mooney DJ. Coating of VEGFreleasing scaffolds with bioactive glass for angiogenesis and bone regeneration. Biomaterials. 2006;27:3249-55.

9. Ding WG, Wei ZX, Liu JB. Reduced local blood supply to the tibial metaphysis is associated with ovariectomy-induced osteoporosis in mice. Connect Tissue Res. 2011;52:25-9.

10. Croucher PI, Vedi S, Motley RJ, Garrahan NJ, Stanton MR, Compston JE. Reduced bone formation in patients with osteoporosis associated with inflammatory bowel disease. Osteoporos Int. 1993;3:236-41.

11. Jing H, Liao L, Su X, Shuai Y, Zhang X, Deng Z, Jin Y. Declining histone acetyltransferase GCN5 represses BMSC-mediated angiogenesis during osteoporosis. FASEB J. 2017;31:4422-33.

12. Hu K, Olsen BR. Osteoblast-derived VEGF regulates osteoblast differentiation and bone formation during bone repair. J Clin Invest. 2016;126:509-26.

13. Villarino AV, Kanno Y, O'Shea JJ. Mechanisms and consequences of Jak-STAT signaling in the immune system. Nat Immunol. 2017;18:374-84.

14. Chim SM, Kuek V, Chow ST, Lim BS, Tickner J, Zhao J, Chung R, Su YW, Zhang G, Erber W, Xian CJ, Rosen V, Xu J. EGFL7 is expressed in bone microenvironment and promotes angiogenesis via ERK, STAT3, and integrin signaling cascades. J Cell Physiol. 2015;230:82-94.

15. Sun G, Wang Z, Ti Y, Wang Y, Wang J, Zhao J, Qian H. STAT3 promotes bone fracture healing by enhancing the FOXP3 expression and the suppressive function of regulatory T cells. APMIS. 2017;125:752-60.

16. Zhang Z, Welte T, Troiano N, Maher SE, Fu XY, Bothwell AL. Osteoporosis with increased osteoclastogenesis in hematopoietic cell-specific STAT3deficient mice. Biochem Biophys Res Commun. 2005;328:800-7.

17. Liu Z, Lei M, Jiang Y, Hao H, Chu L, Xu J, Luo M, Verfaillie CM, Zweier JL, Liu Z. High glucose attenuates VEGF expression in rat multipotent adult progenitor cells in association with inhibition of JAK2/STAT3 signalling. J Cell Mol Med. 2009:13:3427-36.

18. Wang M, Zhang W, Crisostomo P, Markel T, Meldrum KK, Fu XY, Meldrum DR. STAT3 mediates bone marrow mesenchymal stem cell VEGF production. J Mol Cell Cardiol. 2007;42:1009-15.

19. Liu C, Ma R, Wang L, Zhu R, Liu H, Guo Y, Zhao B, Zhao S, Tang J, Li Y, Niu J, Fu M, Zhang D, Gao S. Rehmanniae Radix in osteoporosis: a review of traditional Chinese medicinal uses, phytochemistry, pharmacokinetics and pharmacology. J Ethnopharmacol. 2017;198:351-62.

20. Jiang B, Liu JH, Bao YM, An LJ. Catalpol inhibits apoptosis in hydrogen peroxide-induced PC12 cells by preventing cytochrome c release and inactivating of caspase cascade. Toxicon. 2004;43:53-9.

21. Zhu Y, Wang Y, Jia Y, Xu J, Chai Y. Catalpol promotes the osteogenic differentiation of bone marrow mesenchymal stem cells via the $\mathrm{Wnt} / \beta$ catenin pathway. Stem Cell Res Ther. 2019;10:37.

22. Zheng XW, Yang WT, Chen S, Xu QQ, Shan CS, Zheng GQ, Ruan JC. Neuroprotection of catalpol for experimental acute focal ischemic stroke: preclinical evidence and possible mechanisms of antioxidation, antiinflammation, and antiapoptosis. Oxidative Med Cell Longev. 2017;2017: 5058609 .

23. Dong W, Xian Y, Yuan W, Huifeng Z, Tao W, Zhiqiang L, Shan F, Ya F, Hongli W, Jinghuan W, Lei Q, Li Z, Hongyi Q. Catalpol stimulates VEGF production via the JAK2/STAT3 pathway to improve angiogenesis in rats' stroke model. J Ethnopharmacol. 2016;191:169-79.

24. Meng J, Ma X, Wang N, Jia M, Bi L, Wang Y, Li M, Zhang H, Xue X, Hou Z, Zhou Y, Yu Z, He G, Luo X. Activation of GLP-1 receptor promotes bone marrow stromal cell osteogenic differentiation through $\beta$-catenin. Stem Cell Rep. 2016;6:579-91.

25. Weng SJ, Yan DY, Tang JH, Shen ZJ, Wu ZY, Xie ZJ, Yang JY, Bai BL, Chen L, Boodhun V, Yang L, Da Eric Dong X, Yang L. Combined treatment with Cinnamaldehyde and $\beta$-TCP had an additive effect on bone formation and angiogenesis in critical size calvarial defect in ovariectomized rats. Biomed Pharmacother. 2019;109:573-81.
26. Weng SJ, Yan DY, Gu LJ, Chen L, Xie ZJ, Wu ZY, Tang JH, Shen ZJ, Li H, Bai BL, Boodhun V, Yang L. Combined treatment with vitamin K2 and PTH enhanced bone formation in ovariectomized rats and increased differentiation of osteoblast in vitro. Chem Biol Interact. 2019;300:101-10.

27. Mousaei Ghasroldasht M, Matin MM, Kazemi Mehrjerdi H, Naderi-Meshkin H, Moradi A, Rajabioun M, Alipour F, Ghasemi S, Zare M, Mirahmadi M, Bidkhori HR, Bahrami AR. Application of mesenchymal stem cells to enhance nonunion bone fracture healing. J Biomed Mater Res A. 2019;107:301-11.

28. Ye D, Chen C, Wang Q, Zhang Q, Li S, Liu H. Short-wave enhances mesenchymal stem cell recruitment in fracture healing by increasing HIF-1 in callus. Stem Cell Res Ther. 2020;11:382.

29. Conway JD, Shabtai L, Bauernschub A, Specht SC. BMP-7 versus BMP-2 for the treatment of long bone nonunion. Orthopedics. 2014;37:e1049-57.

30. Granero-Moltó F, Weis JA, Miga MI, Landis B, Myers TJ, O'Rear L, Longobardi $L$, Jansen ED, Mortlock DP, Spagnoli A. Regenerative effects of transplanted mesenchymal stem cells in fracture healing. Stem cells (Dayton, Ohio). 2009; 27:1887-98.

31. Zhang T, Han W, Zhao K, Yang W, Lu X, Jia Y, Qin A, Qian Y. Psoralen accelerates bone fracture healing by activating both osteoclasts and osteoblasts. FASEB J. 2019;33:5399-410.

32. Karvande A, Khedgikar V, Kushwaha P, Ahmad N, Kothari P, Verma A, Kumar P, Nagar GK, Mishra PR, Maurya R, Trivedi R. Heartwood extract from Dalbergia sissoo promotes fracture healing and its application in ovariectomy-induced osteoporotic rats. J Pharm Pharmacol. 2017;69:138197.

33. Udalamaththa VL, Jayasinghe CD, Udagama PV. Potential role of herbal remedies in stem cell therapy: proliferation and differentiation of human mesenchymal stromal cells. Stem Cell Res Ther. 2016;7:110.

34. Cheng J, Xu HY, Liu MM, Cai JP, Wang L, Hua Z, Wu XD, Huo WL, Lv NN Catalpol Promotes the Proliferation and Differentiation of Osteoblasts Induced by High Glucose by Inhibiting KDM7A. Diabetes Metabolic Syndr Obes. 2020;13:705-12.

35. Choi EM, Suh KS, Jung WW, Yun S, Park SY, Chin SO, Rhee SY, Chon S. Catalpol protects against 2,3,7,8-tetrachlorodibenzo-p-dioxin-induced cytotoxicity in osteoblastic MC3T3-E1 cells. J Appl Toxicol. 2019;39:1710-9.

36. Lai N, Zhang J, Ma X, Wang B, Miao X, Wang Z, Guo Y, Wang L, Yao C, Li X, Jiang $G$. Regulatory effect of catalpol on Th1/Th2 cells in mice with bone loss induced by estrogen deficiency. Am J Reprod Immunol (New York, N.Y. : 1989). 2015;74:487-98.

37. Huang $B$, Wang W, Li Q, Wang Z, Yan B, Zhang Z, Wang L, Huang M, Jia C, Lu J, Liu S, Chen H, Li M, Cai D, Jiang Y, Jin D, Bai X. Osteoblasts secrete Cxcl9 to regulate angiogenesis in bone. Nat Commun. 2016;7:13885.

38. Han G, Zheng Z, Pan Z, Lin Y, Gan S, Jiao Y, Li H, Zhou C, Ding S, Li L. Sulfated chitosan coated polylactide membrane enhanced osteogenic and vascularization differentiation in MC3T3-E1s and HUVECS co-cultures system. Carbohydr Polym. 2020;245:116522.

39. Weng SJ, Xie ZJ, Wu ZY, Yan DY, Tang JH, Shen ZJ, Li H, Bai BL, Boodhun V, Eric Dong XD, Yang L. Effects of combined menaquinone-4 and PTH (1-34) treatment on osetogenesis and angiogenesis in calvarial defect in osteopenic rats. Endocrine. 2019;63:376-84.

40. Xie Z, Weng S, Li H, Yu X, Lu S, Huang K, Wu Z, Bai B, Boodhun V, Yang L. Teriparatide promotes healing of critical size femur defect through accelerating angiogenesis and degradation of $\beta$-TCP in OVX osteoporotic rat model. Biomed Pharmacother. 2017;96:960-7.

41. Sims NA. The JAK1/STAT3/SOCS3 axis in bone development, physiology, and pathology. Exp Mol Med. 2020;52:1185-97.

42. Yu X, Li Z, Wan Q, Cheng X, Zhang J, Pathak JL, Li Z. Inhibition of JAK2/ STAT3 signaling suppresses bone marrow stromal cells proliferation and osteogenic differentiation, and impairs bone defect healing. Biol Chem. 2018;399:1313-23.

43. Xu H, Zhou S, Qu R, Yang Y, Gong $X$, Hong $Y$, Jin A, Huang $X$, Dai Q, Jiang L. Icariin prevents oestrogen deficiency-induced alveolar bone loss through promoting osteogenesis via STAT3. Cell Prolif. 2020;53:e12743.

44. Yu X, Wan Q, Ye X, Cheng Y, Pathak JL, Li Z. Cellular hypoxia promotes osteogenic differentiation of mesenchymal stem cells and bone defect healing via STAT3 signaling. Cell Mol Biol Lett. 2019;24:64.

45. Dalagiorgou G, Piperi C, Adamopoulos C, Georgopoulou U, Gargalionis AN, Spyropoulou A, Zoi I, Nokhbehsaim M, Damanaki A, Deschner J, Basdra EK, Papavassiliou AG. Mechanosensor polycystin-1 potentiates differentiation of human osteoblastic cells by upregulating Runx2 expression via induction of JAK2/STAT3 signaling axis. Cell Mol Life Sci. 2017;74:921-36. 
46. Aggarwal BB, Kunnumakkara AB, Harikumar KB, Gupta SR, Tharakan ST, Koca C, Dey S, Sung B. Signal transducer and activator of transcription-3, inflammation, and cancer: how intimate is the relationship? Ann N Y Acad Sci. 2009:1171:59-76.

47. Staines Boone AT, Alcántara-Montiel JC, Sánchez-Sánchez LM, Arce-Cano M, García-Campos J, Lugo Reyes SO. Zoledronate as effective treatment for minimal trauma fractures in a child with STAT3 deficiency and osteonecrosis of the hip. Pediatr Blood Cancer. 2016;63:2054-7.

48. Davidson RK, Himes ER, Takigawa S, Chen A, Horn MR, Meijome T, Wallace $J M$, Kacena MA, Yokota H, Nguyen AV, Li J. The loss of STAT3 in mature osteoclasts has detrimental effects on bone structure. PLoS One. 2020;15: e0236891.

49. Zhou H, Newnum AB, Martin JR, Li P, Nelson MT, Moh A, Fu XY, Yokota H, Li J. Osteoblast/osteocyte-specific inactivation of Stat3 decreases load-driven bone formation and accumulates reactive oxygen species. Bone. 2011;49: 404-11.

50. Xia Y, Ling X, Hu G, Zhu Q, Zhang J, Li Q, Zhao B, Wang Y, Deng Z. Small extracellular vesicles secreted by human iPSC-derived MSC enhance angiogenesis through inhibiting STAT3-dependent autophagy in ischemic stroke. Stem Cell Res Ther. 2020;11:313.

51. Ju X, Xue D, Wang T, Ge B, Zhang Y, Li Z. Catalpol promotes the survival and VEGF secretion of bone marrow-derived stem cells and their role in myocardial repair after myocardial infarction in rats. Cardiovasc Toxicol. 2018;18:471-81.

\section{Publisher's Note}

Springer Nature remains neutral with regard to jurisdictional claims in published maps and institutional affiliations.

Ready to submit your research? Choose BMC and benefit from:

- fast, convenient online submission

- thorough peer review by experienced researchers in your field

- rapid publication on acceptance

- support for research data, including large and complex data types

- gold Open Access which fosters wider collaboration and increased citations

- maximum visibility for your research: over $100 \mathrm{M}$ website views per year

At $\mathrm{BMC}$, research is always in progress.

Learn more biomedcentral.com/submissions 